\title{
miR-217 targeting DKK1 promotes cancer stem cell properties via activation of the Wnt signaling pathway in hepatocellular carcinoma
}

\author{
CHUNLIN JIANG ${ }^{1,2^{*}}$, MIAO YU ${ }^{3 *}$, XIAOYAN XIE ${ }^{1,2}$, GUANGLIANG HUANG $^{1,2}$, YAO PENG $^{5}$, DONG REN $^{6}$, \\ MANXIA LIN ${ }^{1,2}$, BAOXIAN LIU ${ }^{1,2}$, MING LIU ${ }^{1,2}$, WEI WANG ${ }^{1}$ and MING KUANG ${ }^{2,4}$ \\ ${ }^{1}$ Department of Medical Ultrasonics, Institute of Diagnostic and Interventional Ultrasound, \\ ${ }^{2}$ Division of Interventional Ultrasound, ${ }^{3}$ Center for Private Medical Service and Healthcare, \\ Departments of ${ }^{4}$ Liver Surgery, ${ }^{5}$ Gastroenterology and ${ }^{6}$ Orthopaedic Surgery/Orthopaedic Research Institute, \\ The First Affiliated Hospital of Sun Yat-sen University, Guangzhou, Guangdong 510080, P.R. China
}

Received November 8, 2016; Accepted May 24, 2017

DOI: $10.3892 /$ or.2017.5924

\begin{abstract}
Hepatocellular carcinoma (HCC) is one of the most common malignancies and exhibits heterogeneity in terms of clinical outcomes and biological activities. Emerging evidence has demonstrated that cancer stem cells (CSCs) play important roles in the tumorigenesis and progression of HCC. However, the molecular mechanisms underlying the stemness maintenance of CSCs remain largely unknown. In the present study, through real-time PCR, western blotting, luciferase assays, RNA immunoprecipitation, and in vitro and in vivo assays, we demonstrated that miR-217 expression was markedly increased in HCC tissues and cells. Overexpression of miR-217 promoted, while silencing miR-217 suppressed, the fraction of the side population and the expression of cancer stem cell factors in vitro and tumorigenicity in vivo in HCC cells. Our findings further demonstrated that miR-217 promoted the CSC-like phenotype via dickkopf-1 (DKK1) targeting, resulting in constitutive activation of Wnt signaling. Moreover, the stimulatory effects of miR-217 on stem cell properties and Wnt signaling were antagonized by the upregulation of DKK1 in miR-217-overexpressing cells. Conversely, the inhibitory effects of silencing miR-217 on stem cell properties and Wnt signaling were reversed by the downregulation of DKK1 in miR-217-downregulated cells. Therefore, our results indicate that miR-217 plays a vital role in the CSC-like phenotypes of HCC cells and may be used as a potential therapeutic target against HCC.
\end{abstract}

Correspondence to: Dr Ming Kuang, Department of Interventional Ultrasound/Liver Surgery, The First Affiliated Hospital of Sun Yat-sen University, 58 Zhong Shan Road 2, Guangzhou, Guangdong 510080, P.R. China

E-mail: kuangm@mail.sysu.edu.cn

*Contributed equally

Key words: miR-217, cancer stem cells, DKK1, Wnt signaling pathway, hepatocellular carcinoma

\section{Introduction}

Hepatocellular carcinoma (HCC) is the fifth most prevalent cancer and the third-leading cause of cancer-related deaths worldwide (1). Despite great progress in systemic management and treatment in recent years, the 5-year survival rate is still poor, $\sim 15 \%$, where locoregional and distant recurrences remain primary issues due to widespread intrahepatic and extrahepatic metastases (2). Therefore, it is of great urgency to explore the molecular mechanisms responsible for HCC development and progression, which may help to identify new antitumor strategies against HCC.

Cancer stem cells (CSCs) are a minority cell population within tumors and are characterized by unlimited proliferation as well as the abilities of self-renewal and differentiation into heterogeneous lineages of cancer cells that constitute the major tumor population $(3,4)$. Emerging evidence has demonstrated that CSCs play a crucial role in the progression of different types of cancer $(5,6)$. The existence of CSCs was first reported in acute myeloid leukemia (7), and broad identification was further demonstrated in several solid tumors, including colon (8), pancreas (9) and breast cancer (10), indicating the ubiquitous existence of CSCs in tumors. Furthermore, increasing evidence has suggested that CSCs are the critical initiators of $\mathrm{HCC}$. Lee et al reported that $\mathrm{CD} 24^{+}$cells drive tumor initiation through STAT3-mediated NANOG regulation in HCC (11). Moreover, a monoclonal antibody against CSCs, 1B50-1, decreased self-renewal and tumor formation capacities and induced apoptosis of CSCs in HCC (12). This evidence indicated that therapeutic methods targeting CSCs may be efficient avenues in the treatment of HCC.

MicroRNAs (miRNAs) are non-coding 17- to 25-nucleotide-long RNAs that function by binding to the 3'-untranslated (3'UTR) region of downstream mRNAs and regulate gene expression post-transcriptionally (13). Physiologically, miRNAs play important roles in various cellular functions, including cell apoptosis, proliferation and differentiation (14). Furthermore, miRNAs also play a pivotal role in the stemness maintenance of CSCs, including HCC $(15,16)$. For example, 
Ma et al reported that microRNA expression profiling of $\mathrm{CD} 33^{+}$and $\mathrm{CD} 133^{-}$cells from human $\mathrm{HCC}$ clinical specimens and cell lines identified an overexpression of miR-130b in CD133 ${ }^{+}$CSCs. Ectopic expression of miR-130b in CD133 cells enhanced self-renewal ability and tumorigenicity in vivo. Conversely, antagonizing miR-130b in $\mathrm{CD} 133^{+} \mathrm{CSCs}$ exhibited an opposing effect (17). In addition, another study indicated that miR-181, which was found to be overexpressed in $\mathrm{EpCAM}^{+} \mathrm{HCC}$ cells isolated from $\mathrm{AFP}^{+}$tumors, promoted tumor-initiating ability. Notably, inhibition of miR-181 led to a decrease in tumor-initiating ability (18). Therefore, these studies demonstrated that miRNAs play important roles in regulating CSC-like phenotypes in HCC.

In the present study, we found that miR-217 was upregulated in HCC tissue samples and cells. Moreover, upregulation of miR-217 enhanced the stem cell properties of HCC cells via DKK1 targeting. Notably, the pro-CSC-like phenotype role of miR-217 was attenuated by overexpression of dickkopf-1 (DKK1) in HCC cells. Thus, our results revealed a novel mechanism by which miR-217 promoted a CSC-like phenotype in HCC, and anti-HCC therapy targeting miR-217 may be a potential therapeutic strategy for the treatment of HCC.

\section{Materials and methods}

Cell lines and cell culture. The human HCC cell lines 97H, HepG2, QGY-7703, Hep3B, PLC, Huh7 and SMMC7721 were obtained from the American Type Culture Collection (ATCC; Manassas, VA, USA), and all human HCC cell lines were cultured as described in the ATCC protocol. Human liver immortal cell line L02 was purchased from Biomics Biotechnologies (Nantong, China). The cell lines were maintained in RPMI-1640 medium (Invitrogen, Carlsbad, CA, USA) supplemented with $10 \%$ fetal bovine serum (FBS) (HyClone, Logan, UT, USA) and $100 \mathrm{U} / \mathrm{ml}$ penicillin plus $100 \mu \mathrm{g} / \mathrm{ml}$ streptomycin. The cells were grown in a humidified atmosphere of $5 \% \mathrm{CO}_{2}$ at $37^{\circ} \mathrm{C}$.

Plasmids, transfection and generation of stable cell lines. The miR-217-expression plasmid was generated by cloning the genomic pre-miR-217 gene, with a 300-bp sequence on each flanking side, into retroviral-transfer plasmid pMSCV-puro (Clontech Laboratories, Inc., Mounatin View, CA, USA) to generate plasmid pMSCV-miR-217. pMSCV-miR-217 was cotransfected with the pIK packaging plasmid in 293FT cells using the standard calcium phosphate transfection method, as previously described (19). Thirty-six hours after the co-transfection, supernatants were collected and incubated with cells that were to be infected for $24 \mathrm{~h}$ in the presence of Polybrene $(2.5 \mu \mathrm{g} / \mathrm{ml})$. After infection, puromycin $(1.5 \mu / \mathrm{ml})$ was used to select stably transduced cells over a 10-day period. The 3'UTR of DKK1 was amplified and cloned downstream to the luciferase gene in a modified pGL3 control vector. The reporter plasmids containing wild-type (CCTTTGATC; TOPflash) or mutated (CCTTTGGCC; FOPflash) TCF/LEF DNA binding sites were purchased from Upstate Biotechnology. AntagomiR-217, small interfering RNA (siRNA) for DKK1 knockdown, overexpressing DKK1 plasmid and respective control RNA were synthesized and purified by RiboBio Co., Ltd. (Guangzhou, China). Transfection of miRNAs, siRNAs
Table I. Probe sequences used for real-time PCR.

\begin{tabular}{lll}
\hline Name & & \multicolumn{2}{c}{ Sequence (5' to 3') } \\
\hline DKK1 & F & TTTCCTCAATTTCTCCTCGG \\
& R & ATGCGTCACGCTATGTGCT \\
c-Myc & F & CACCGAGTCGTAGTCGAGGT \\
& R & TTTCGGGTAGTGGAAAACCA \\
BMI1 & F & TCGTTGTTCGATGCATTTCT \\
& R & CTTTCATTGTCTTTCCGCC \\
OCT4 & F & GGTTCTCGATACTGGTTCGC \\
& R & GTGGAGGAAGCTGACAACAA \\
Nanog & F & ATGGAGGAGGGAAGAGGAGA \\
Sox2 & R & GATTTGTGGGCCTGAAGAAA \\
& F & GCTTAGCCTCGTCGATGAAC \\
TCF1 & R & AACCCCAAGATGCACAACTC \\
& F & GACTTGACCATCTTCGCCAC \\
LEF1 & R & CCTCAAAGAGCTGGAGAACCT \\
& F & CACTGTAAGTGATGAGGGGG \\
GAPDH & R & TGGATCTCTTTCTCCACCCA \\
& F & AGAGGCAGGGATGATGTTCTG \\
& R & AGAGGCAGGGATGATGTTCTG \\
& &
\end{tabular}

F, forward; R, reverse.

and plasmids was performed using Lipofectamine 3000 (Life Technologies, Grand Island, NY, USA) according to the manufacturer's instructions.

RNA extraction, reverse transcription and real-time RT-PCR. Total RNA was extracted from cultured cells using the mirVana miRNA Isolation kit (Ambion Life Technologies, Carlsbad,CA, USA). cDNA was synthesized from total RNA with specific stem-loop primers and the TaqMan MicroRNA Reverse Transcription kit (Applied Biosystems Life Technologies, Foster City, CA, USA). The expression of miRNAs was analyzed by real-time PCR using the TaqMan MicroRNA Assay kit (Applied Biosystems Life Technologies). Detection of mRNA was performed as previously described (20). The sequences of the primers are listed in Table I.

Patients and tumor tissues. A total of 58 paraffin-embedded, archived separate HCC tissues and 6 paired HCC tissues with the matched adjacent normal tissues were obtained during surgery at the First Affiliated Hospital of Sun Yat-sen University (Guangzhou, China) between 2006 and 2010. Patients were diagnosed based on clinical and pathological evidence, and the specimens were immediately snap-frozen and stored in liquid nitrogen tanks. For the use of these clinical materials for research purposes, prior informed patient consent and approval from the Institutional Research Ethics Committee were obtained.

Western blotting. Western blotting was performed according to a standard method, as previously described (21). The following primary antibodies were used: anti-DKK1, anti- $\beta$-catenin, 
and $\alpha$-tubulin (BD Pharmingen; BD Biosciences, San Diego, CA, USA) and p84 (Abcam, Cambridge, MA, USA). Nuclear extracts were prepared using the Nuclear Extraction kit (Active Motif, Carlsbad, CA, USA), according to the manufacturer's instructions.

Luciferase reporter assay. Cells were seeded in triplicate in 24 -well plates, and allowed to settle for $24 \mathrm{~h}$. Indicated plasmids plus $1 \mathrm{ng}$ pRL-TK Renilla plasmid were transfected into the cells using Lipofectamine 3000 reagent (Life Technologies). Forty-eight hours after transfection, a Dual-Luciferase Reporter Assay (Promega, Madison, WI, USA) was performed according to the manufacturer's instructions, as previously described (22).

Tumor xenografts. All experimental procedures were approved by the Institutional Animal Care and Use Committee of Sun Yat-sen University. Six-week-old BALB/c-nu mice were randomly divided into 4 groups (n=6/group). The cells $\left(1 \times 10^{5}\right)$ were subcutaneously inoculated along with Matrigel (final concentration of $25 \%$ ) into the inguinal folds of the nude mice. The tumor volume was determined using an external caliper and calculated using the equation $\left(\mathrm{L} \mathrm{x} \mathrm{W}^{2}\right) / 2$. The mice were sacrificed 35 days after inoculation and the tumors were excised and subjected to pathological examination.

Side population analysis. The cell suspensions were labeled with Hoechst 33342 (molecular probes, \#H-3570) dye for side population (SP) analysis as per standard protocol (23). Briefly, cells were resuspended at $1 \mathrm{X}$ pre-warmed Opti-MEM (containing 2\% FBS) (both from Gibco, Grand Island, NY, USA) at a density of $10^{6}$ cells $/ \mathrm{ml}$. Hoechst 33342 dye was added at a final concentration of $5 \mu \mathrm{g} / \mathrm{ml}$ in the presence or absence of verapamil (50 $\mu \mathrm{mol} / \mathrm{l}$; Sigma-Aldrich, St. Louis, MO, USA) and the cells were incubated at $37^{\circ} \mathrm{C}$ for $90 \mathrm{~min}$ with intermittent shaking. At the end of the incubation period, the cells were washed with Opti-MEM containing $2 \%$ FBS and centrifuged at $4^{\circ} \mathrm{C}$, and subsequently resuspended in ice-cold Opti-MEM containing $2 \% \mathrm{FBS}$ and $10 \mathrm{mmol} / \mathrm{l}$ HEPES. Then, propidium iodide (Sigma-Aldrich) at a final concentration of $2 \mu \mathrm{g} / \mathrm{ml}$ was added to the cells to gate viable cells. The cells were filtered through a $40-\mu \mathrm{m}$ cell strainer to obtain a single cell suspension before sorting. Analysis and sorting was carried out on a FACSAria I (Becton-Dickinson, Franklin Lakes, NJ, USA). The Hoechst 33342 dye was excited at $355 \mathrm{~nm}$ and its dual-wavelength emission at the blue and red regions was plotted to get the SP scatter.

miRNP immunoprecipitation. Cells were co-transfected with HA-Ago1 along with $100 \mathrm{nM}$ miR-217, followed by HA-Agol immunoprecipitation using an HA-antibody. Real-time PCR analysis of the IP material was used to assess the association of the mRNA of DKK1 with the RISC complex.

Statistical analysis. All statistical analyses were carried out using the SPSS 16.0 statistical software package. Comparisons between groups for statistical significance were performed using Chi-square and Fisher's exact tests. In all cases, $\mathrm{P}<0.05$ was considered significant. All the experiments were repeated 3 times.
Table II. Relationship between miR-217 and the clinicopathological characteristics in 64 hepatocellular carcinoma patients.

\begin{tabular}{|c|c|c|c|c|}
\hline \multirow[b]{2}{*}{ Parameters } & \multirow[b]{2}{*}{ No. of cases } & \multicolumn{2}{|c|}{$\begin{array}{c}\text { miR-217 } \\
\text { expression }\end{array}$} & \multirow[b]{2}{*}{ P-values } \\
\hline & & High & Low & \\
\hline Gender & & & & 0.448 \\
\hline Male & 37 & 20 & 17 & \\
\hline Female & 27 & 12 & 15 & \\
\hline Age (years) & & & & 0.211 \\
\hline$<60$ & 33 & 14 & 19 & \\
\hline$\geq 60$ & 31 & 18 & 13 & \\
\hline $\operatorname{AFP}(\mathrm{ng} / \mathrm{ml})$ & & & & $0.003^{\mathrm{a}}$ \\
\hline$<400$ & 30 & 9 & 21 & \\
\hline$\geq 400$ & 34 & 23 & 11 & \\
\hline Tumor size $(\mathrm{cm})$ & & & & $<0.001^{\mathrm{a}}$ \\
\hline$<5$ & 38 & 11 & 27 & \\
\hline$\geq 5$ & 26 & 21 & 5 & \\
\hline Differentiation & & & & 0.080 \\
\hline High & 33 & 20 & 13 & \\
\hline Low & 31 & 12 & 19 & \\
\hline Clinical stage & & & & $0.045^{\mathrm{a}}$ \\
\hline I-II & 30 & 11 & 19 & \\
\hline III-IV & 34 & 21 & 13 & \\
\hline Venous invasion & & & & $0.042^{\mathrm{a}}$ \\
\hline Negative & 26 & 9 & 17 & \\
\hline Positive & 38 & 23 & 15 & \\
\hline Distant metastasis & & & & $0.006^{\mathrm{a}}$ \\
\hline Negative & 45 & 8 & 19 & \\
\hline Positive & 35 & 24 & 13 & \\
\hline
\end{tabular}

${ }^{\mathrm{a}} \mathrm{P}<0.05$. AFP, $\alpha$-fetoprotein.

\section{Results}

miR-217 is upregulated in HCC tissues and cells. To identify the potential miRNAs in HCC, we analyzed the HCC datasets from The Cancer Genome Atlas (TCGA). The result revealed that miR-217 was upregulated in HCC tissues compared with normal hepatic tissues (Fig. 1A). We further analyzed the miR-217 expression in 49 paired HCC tissues from TCGA and found that the expression levels of miR-217 were upregulated in the primary HCC tissues compared with the matched adjacent normal tissues (Fig. 1B). Furthermore, we assessed the miR-217 expression in our own HCC tissue samples (Table II). As shown in Fig. 1C and D, miR-217 expression was markedly increased in HCC tissues compared with that in 6 normal hepatic tissues, and the matched adjacent normal tissues, respectively. We further examined the expression level of miR-217 in HCC cells and found that compared to human liver immortal cell line L02, the expression of miR-217 was differentially upregulated in HCC cells. Thus, our findings demonstrated that miR-217 is increased in HCC tissues and cells. 

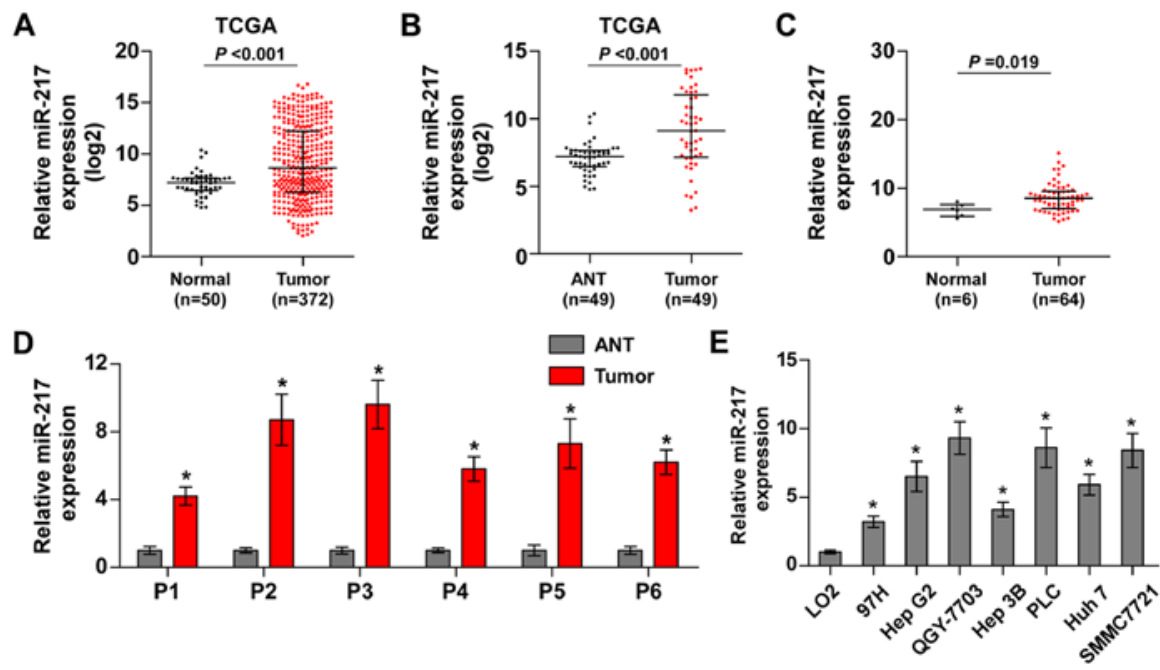

Figure 1. miR-217 is upregulated in HCC tissues and cells (A) The expression level of miR-217 in normal hepatic tissues and HCC tissues in the miRNA sequencing dataset of TCGA HCC (normal, $n=50$; hepatocellular carcinoma, $n=372$ ). $\mathrm{P}<0.001$. (B) The expression level of miR-217 in 49 paired primary HCC tissues compared with matched adjacent normal tissues. The transcript levels were normalized to U6 expression. P $<0.001$. (C) Real-time PCR analysis of miR-217 expression in $64 \mathrm{HCC}$ tissues compared to 6 normal hepatic tissues. The transcript levels were normalized to U6 expression. P<0.05. (D) The expression level of miR-217 in 6 paired primary HCC tissues compared with matched adjacent normal tissues. The transcript levels were normalized to U6 expression. ${ }^{*} \mathrm{P}<0.05$. (E) The expression level of miR-217 in one normal hepatic cell line and 7 HCC cell lines. ${ }^{*} \mathrm{P}<0.05$. TCGA, The Cancer Genome Atlas; HCC, hepatocellular carcinoma.

A

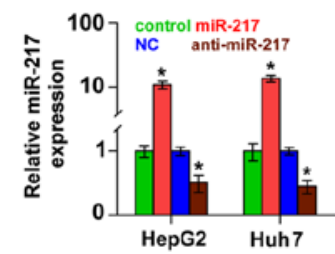

B

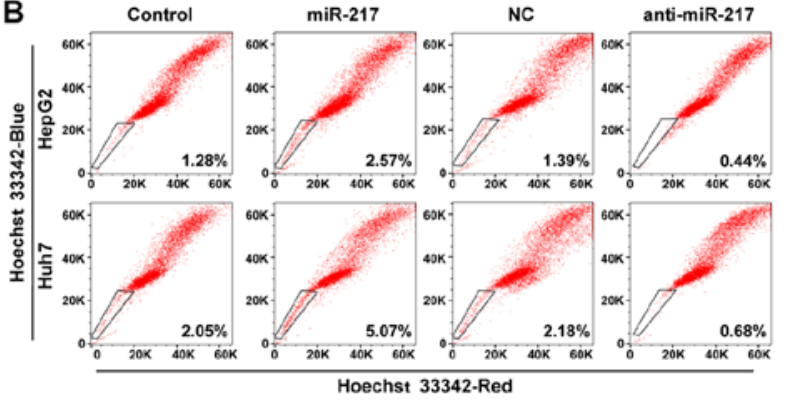

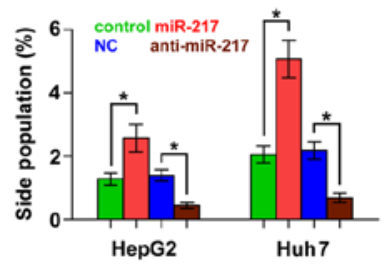

C

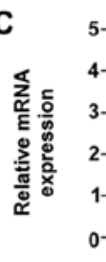

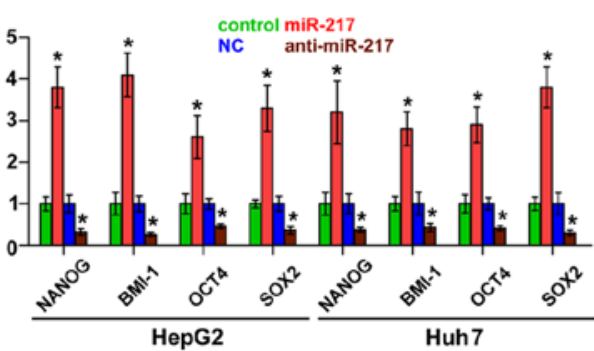

E

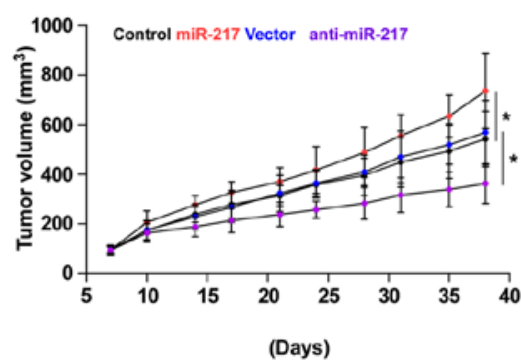

D

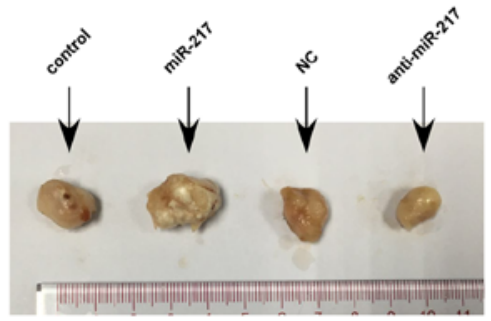

$\mathbf{F}$

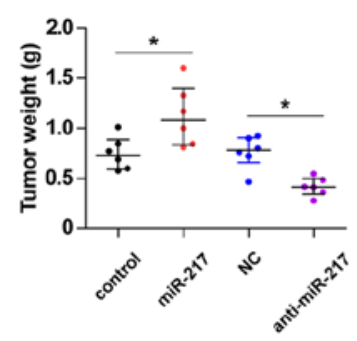

Figure 2. miR-217 promotes the CSC population of HCC in vitro and in vivo. (A) Real-time PCR analysis of miR-217 expression after transfection with miR-217 mimics or inhibitor in HCC cells. (B) Hoechst 33342 dye exclusion assay showing that upregulation of miR-217 increased the side population, whereas silencing of miR-217 decreased the side population. Error bars represent the mean \pm SD of three independent experiments. ${ }^{*} \mathrm{P}<0.05$. (C) Real-time PCR analysis of NANOG, BMI-1, OCT4 and SOX2 expression. GAPDH was used as the loading control. Error bars represent the mean \pm SD of 3 independent experiments. ${ }^{*} \mathrm{P}<0.05$. (D) Representative images of the tumors are shown in the xenograft model of nude mice. Tumors formed by the miR-217-overexpressing Huh7 cells were larger than the control tumors. Conversely, tumors formed by the anti-miR-217 cells were smaller than tumors formed by the NC vector cells. (E) Growth curves for tumor formation after implantation ( $\mathrm{n}=6 /$ group). Mean tumor volumes are plotted. ${ }^{*} \mathrm{P}<0.05$. (F) A dot plot displaying the mean tumor weights of each group ( $\mathrm{n}=6 /$ group). ${ }^{*} \mathrm{P}<0.05$. CSC, cancer stem cell; HCC, hepatocellular carcinoma. 
A

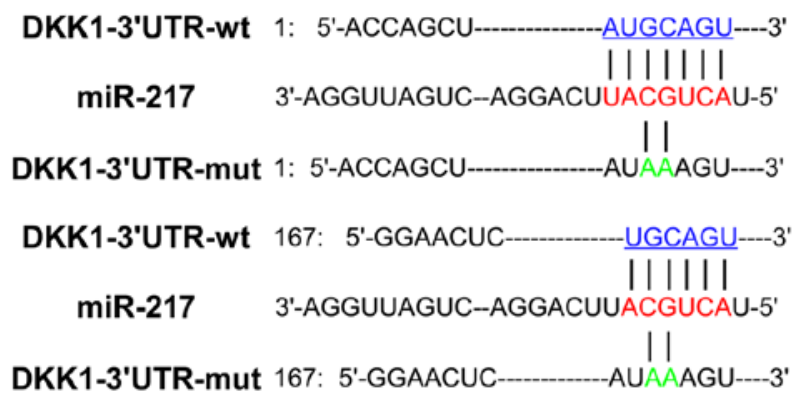

B

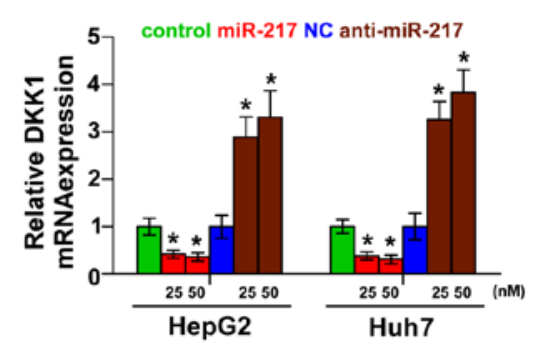

C

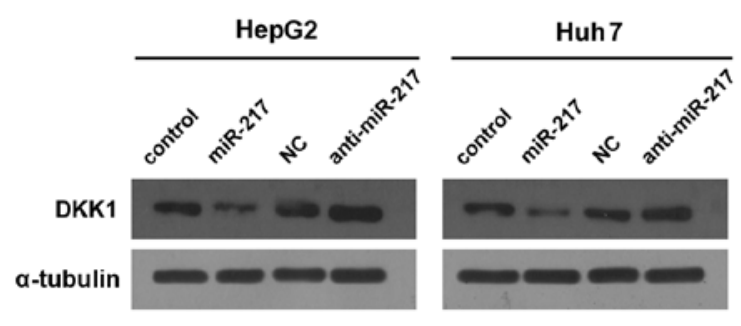

E

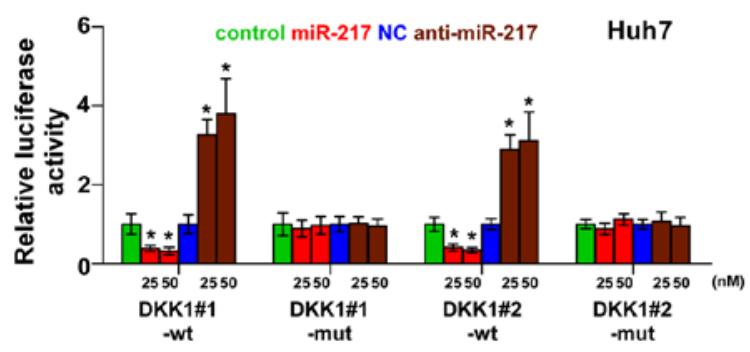

D

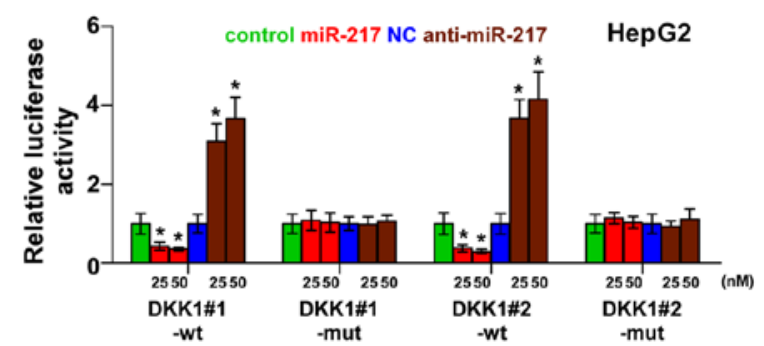

$\mathbf{F}$

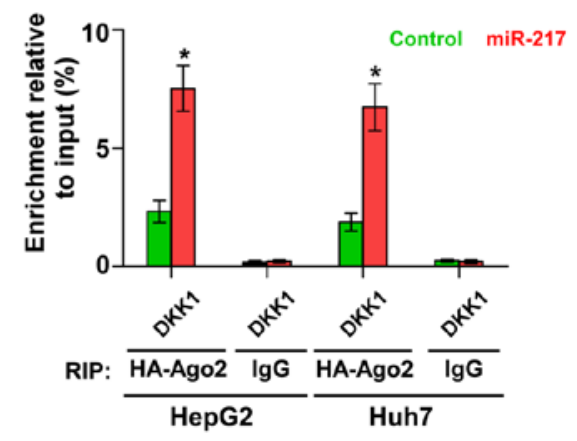

Figure 3. miR-217 targets DKK1 in HCC cells. (A) Predicted miR-217 target sequence in the 3 'UTRs of DKK1. (B) Real-time PCR analysis of DKK1 expression. GADPH served as the loading control. Error bars represent the mean \pm SD of 3 independent experiments. "P $<0.05$. (C) Western blotting of DKK1 expression. $\alpha$-tubulin served as the loading control. (D and E) Luciferase assay of the indicated cells transfected with pmirGLO-3'UTR reporter in miR217-overexpressing and -silenced cells. Error bars represent the mean $\pm \mathrm{SD}$ of 3 independent experiments. ${ }^{*} \mathrm{P}<0.05$. (F) miRNP IP assay showing the association between miR-217 and the DKK1 transcript in HCC cells. Pull-down of the IgG antibody served as the negative control. Error bars represent the mean \pm SD of 3 independent experiments. "P<0.05. DKK1, dickkopf-1, HCC, hepatocellular carcinoma.

miR-217 promotes stem cell properties and tumorigenesis in HCC cells. Accumulating studies have suggested that cancer growth is driven by CSCs $(24,25)$. To further investigate the role of miR-217 in the CSC-like phenotype of HCC cells, we first constructed miR-217-stably expressing cells by ectopically overexpressing miR-217 and endogenously silencing miR-217 via retrovirus infection in the HepG2 and Huh7 cell lines (Fig. 2A). The effect of miR-217 on the SP was examined and the result revealed that overexpression of miR-217 increased, while silencing miR-217 decreased the fraction of SP cells in the HepG2 and Huh7 cells (Fig. 2B). We further assessed the effect of miR-217 on stem cell markers, including Nanog homeobox (NANOG), proto-oncogene polycomb ring finger (BMI1), POU class 5 homeobox 1A (OCT4A) and SOX2, and found that upregulation of miR-217 enhanced the expression of these markers. However, knockdown of miR-217 decreased the expression of these markers (Fig. 2C). Collectively, these results indicated that miR-217 promotes CSC-like phenotypes in HCC in vitro.
We next assessed the effect of miR-217 on the tumorigenesis of HCC in vivo. Huh7 cells $\left(1 \times 10^{5}\right)$ of the miR-217-overexpressing, control and anti-miR-217 cells were inoculated into nude mice, respectively. As shown in Fig. 2D-F, tumors in the miR-217-overexpressing cells grew more rapidly than those in the control group. Conversely, tumors in the anti-miR-217 group were smaller than the tumors in the negative control group. These results revealed that miR-217 promotes the tumorigenesis of HCC cells in vivo.

miR-217 targets a negative regulator of the Wnt signaling pathway. By the publicly available algorithms TargetScan and miRNA.ORG, we found that the negative regulator of Wnt signaling, DKK1, may be a potential target of miR-217 (Fig. 3A). PCR and western blotting revealed that miR-217 overexpression decreased the mRNA and protein expression levels of DKK1. Conversely, silencing of miR-217 increased the expression of DKK1 (Fig. 3B and C). Luciferase assay revealed that miR-217 overexpression decreased, while 
A

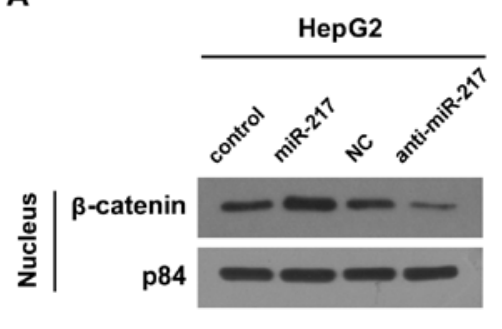

C

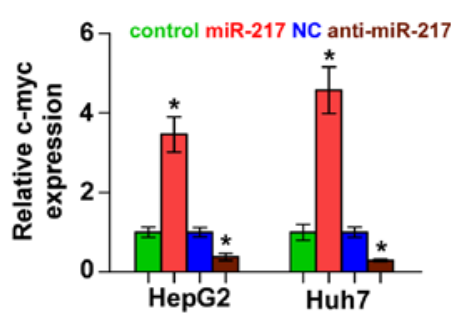

B

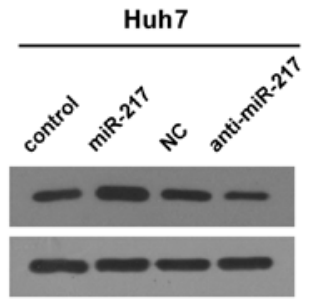

D

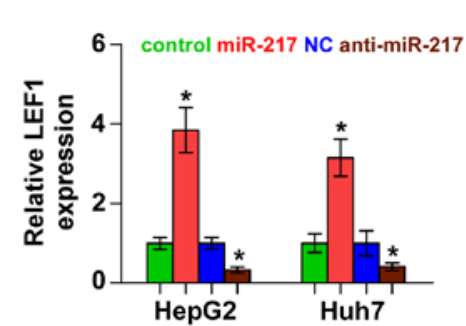

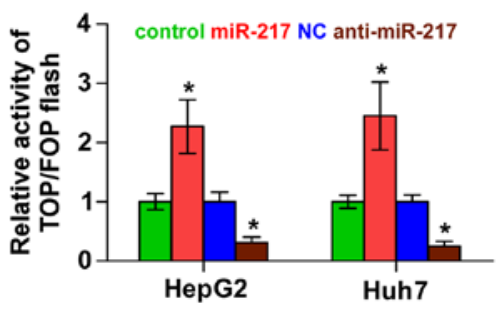

E

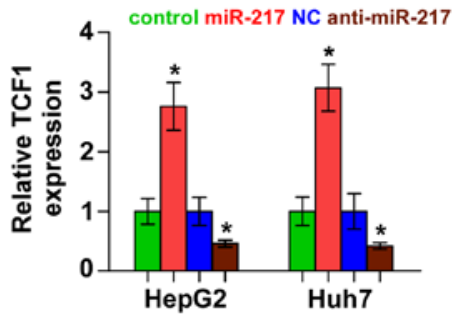

Figure 4. miR-217 activates the Wnt signaling pathway. (A) Western blotting of nuclear $\beta$-catenin expression. The nuclear protein p84 was used as the nuclear protein marker. (B) TOP/FOP luciferase assay of TCF/LEF transcriptional activity in the indicated cells. Error bars represent the mean \pm SD of 3 independent experiments. ${ }^{*} \mathrm{P}<0.05$. (C-E) Real-time PCR analysis of c-myc, LEF1 and TCF1 expression in the indicated cells. ${ }^{*} \mathrm{P}<0.05$.

A

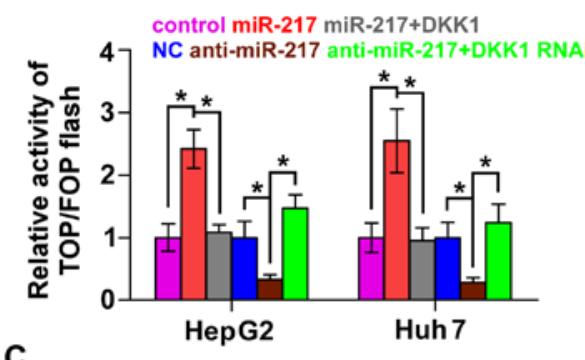

B

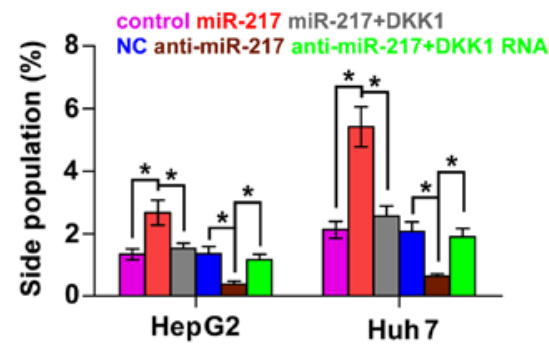

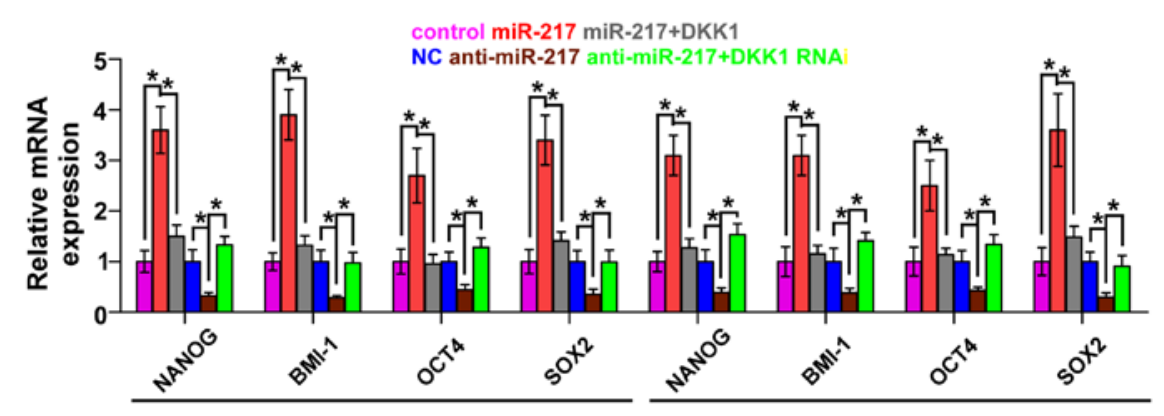

Figure 5. DKK1 mediates miR-217-induced and Wnt signaling activity and CSC phenotypes in HCC cells. (A) Upregulation or silencing of DKK1 reversed the TCF/LEF transcriptional activity in HCC cells. Error bars represent the mean $\pm \mathrm{SD}$ of 3 independent experiments. " $\mathrm{P}<0.05$. (B) Upregulation or silencing of DKK1 reversed the fraction of the side population in HCC cells. Error bars represent the mean \pm SD of 3 independent experiments. * $\mathrm{P}<0.05$. (C) Upregulation or silencing of DKK1 reversed the expression levels of NANOG, BMI-1, OCT4 and SOX2 in HCC cells. Error bars represent the mean \pm SD of 3 independent experiments. " $\mathrm{P}<0.05$. CSC, cancer stem cell; DKK1, dickkopf-1; HCC, hepatocellular carcinoma.

silencing of miR-217 enhanced the reporter activity driven by the 3'UTRs of DKK1 in dose-dependent manners, but not by the mutant 3'UTRs of DKK1 within the miR-217-binding seed regions in HCC cells (Fig. 3D and E). Furthermore, microribonucleoprotein (miRNP) immunoprecipitation (IP) assay demonstrated a selective association of miR-217 with DKK1 transcript (Fig. 3F), further elucidating the direct suppressive effect of miR-217 on DKK1. Consequently, our results indicate that DKK1 is an authentic target of miR-217 in HCC cells.
miR-217 activates Wnt signaling pathways in HCC cells. We next investigated the effect of miR-217 on Wnt signaling. As shown in Fig. 4A, western blotting revealed that overexpression of miR-217 increased nuclear accumulation of $\beta$-catenin, while silencing of miR-217 impaired the $\beta$-catenin translocation into the nucleus. Furthermore, we found that miR-217 overexpression increased, while silencing of miR-217 decreased, $\beta$-catenin/TCF transcriptional activity (Fig. 4B). We further examined the expression levels of multiple downstream genes of Wnt signaling, including c-myc, LEF1 and TCF1 and found 
A

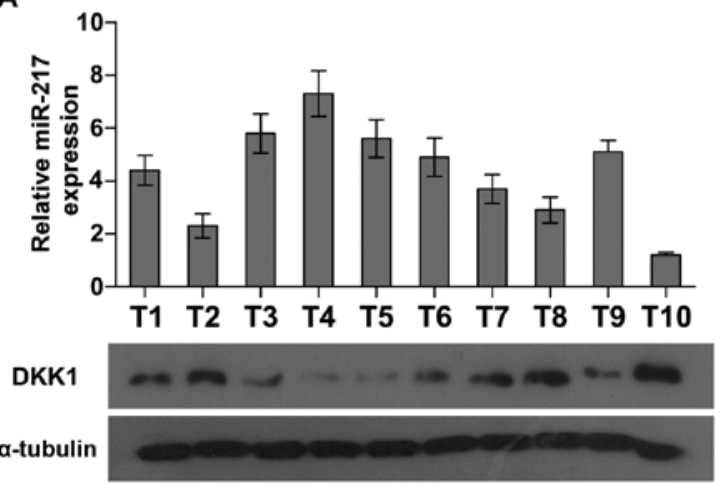

B

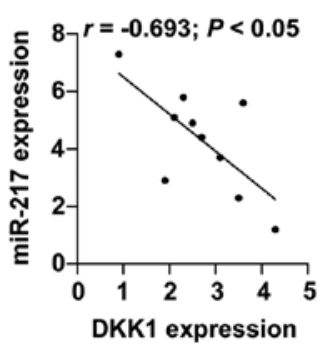

C

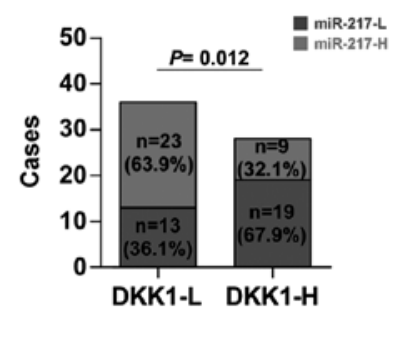

Figure 6. Expression of miR-217 is inversely correlated with DKK1 expression in HCC clinical tissues. (A) Real-time PCR of miR-217 expression in 10 fresh HCC tissues. U6 was used as the loading control. Each bar represents the mean \pm SD of 3 independent experiments. Western blotting of DKK1 expression in 10 fresh HCC tissues. $\alpha$-tubulin was used as the loading control. (B) Correlation between the expression of miR-217 and DKK1 in HCC clinical tissues. The expression level of DKK1 was quantified by densitometry using Quantity One Software, and normalized to the level of $\alpha$-tubulin. (C) miR-217 was increased in HCC tissues with low DKK1 expression compared to those with high DKK1 expression. DKK1, dickkopf-1; HCC, hepatocellular carcinoma.

that overexpression of miR-217 enhanced the expression of c-myc, LEF1 and TCF1, while silencing of miR-217 yielded the opposite effect (Fig. 4C-E). Thus, these results demonstrated that miR-217 activates Wnt signaling pathways in HCC cells.

DKK1 mediates miR-217-induced CSC phenotypes in HCC cells. To explore whether DKK1 contributed to miR-217-induced CSCs, we transfected the DKK1 plasmid in miR-217-overexpressing cells and RNA interference of DKK1 in miR-217-silenced cells, respectively. $\beta$-catenin/TCF transcriptional activity assay revealed that the stimulatory effect of miR-217 was angatonized by the upregulation of DKK1 and the inhibitory effect of anti-miR-217 was reversed by DKK1 RNAi (Fig. 5A). Furthermore, the upregulation of DKK1 in miR-217-overexpressing cells significantly decreased the SP. In contrast, knockdown of DKK1 in miR-217-silenced cells increased the fraction of SP in HCC cells (Fig. 5B). Real-time PCR revealed that DKK1 contributed to the expression of stem cell markers regulated by miR-217 (Fig. 5C). Collectively, these results suggest that DKK1 mediates the regulatory role of miR-217 in CSC-like phenotypes in HCC cells.

Clinical correlation of miR-217 with DKK1 expression in HCC tissues. To further investigate whether the expression of miR-217 was associated with DKK1 expression in HCC clinical tissue samples, the expression levels of miR-217 were examined in $10 \mathrm{HCC}$ fresh tissues by real-time PCR. As shown in Fig. 6A and $\mathrm{B}$, there was a significant negative correlation between miR-217 and DKK1 $(r=-0.693$; $\mathrm{P}<0.05)$ in HCC. Furthermore, we found that the expression of miR-217 was increased in HCC tissues with low DKK1 expression when compared to those with high DKK1 expression (Fig. 6C). Collectively, these results demonstrated that miR-217 is negatively correlated with DKK1 expression in HCC tissues.

\section{Discussion}

In the canonical Wnt pathway, Wnt ligands bind to frizzled receptors and lipoprotein receptor-related protein -5 or -6 (LRP5/6) co-receptors, giving rise to the accumulation and subsequent translocation of $\beta$-catenin into the nucleus. Then, nuclear $\beta$-catenin interacts with the T-cell factor (TCF) family of transcription factors to initiate the transcription of downstream target genes $(26,27)$. Notably, several cancer stem cell factors, including CD44, NANOG, SOX2 and OCT4, are well-known targets of the Wnt pathway, indicating that the Wnt signaling pathway plays crucial roles in the regulation of CSCs $(28,29)$. Furthermore, the constitutive activation of $\mathrm{Wnt} / \beta$-catenin signaling has been implicated in a variety of human cancers, including hepatocellular carcinoma (HCC) $(30,31)$. Indeed, $40 \sim 70 \%$ of HCC has been reported to harbor nuclear accumulation of the downstream effector $\beta$-catenin of Wnt signaling (32). Wong et al reported that constitutive activation of Wnt signaling induced the increased translocation of $\beta$-catenin into the nucleus, which contributed to the development of HCC (33). These studies demonstrated that dysregulation of Wnt signaling is a critical driver of HCC pathogenesis. In the present study, we revealed that miR-217 was increased in HCC tissues and cells. Overexpression of miR-217 increased, while silencing of miR-217 decreased the nuclear translocation of $\beta$-catenin, $\beta$-catenin/TCF transcriptional activity as well as the expression levels of downstream genes of the Wnt signaling pathway, which further contributed to the CSC-like phenotype in HCC cells. Our results further revealed that miR-217 activates the Wnt signaling pathway via DKK1 targeting, an important negative regulator of Wnt signaling. Therefore, our findings uncovered a novel mechanism of miR-217 in the regulation of the Wnt signaling pathway.

Dickkopf-1 (DKK1) which acts as an important negative regulator of Wnt/ $\beta$-catenin signaling functions via binding with high affinity to LRP5/6 in competition with Wnt (34). DKK1 has been reported to be downregulated in various cancers, resulting in the constitutive activation of the Wnt/ $\beta$-catenin signaling pathway $(35,36)$. Furthermore, several studies have reported that the upregulation of DKK1 inhibited sphere formation capacity and decreased the $\mathrm{CD} 24^{-} / \mathrm{CD} 44^{+}$cell population in breast cancer cells (37). This evidence indicated that DKK1 played an important role in the stemness maintenance of CSCs. In the present study, RT-PCR and western blotting revealed that overexpressing miR-217 decreased, while silencing miR-217 increased the mRNA and protein levels of DKK1. Luciferase and miRNP IP assay demonstrated the 
association of miR-217 with DKK1 in HCC cells, indicating that DKK1 is a direct target of miR-217 in HCC cells. Notably, the stimulatory effects of miR-217 on $\beta$-catenin/TCF transcriptional activity and CSC-like phenotype was antagonized by overexpression of DKK1 in miR-217-overexpressing cells. Conversely, silencing of DKK1 yielded the opposite effect in miR-217-downregulating cells. Collectively, these findings demonstrated that miR-217 promotes a CSC-like phenotype and activates Wnt signaling via DKK1 targeting in HCC cells.

miR-217 has been identified to be downregulated in multiple human cancers, and has contributed to cancer cell proliferation, drug resistance and metastasis via varying mechanisms (38-41). Furthermore, the expression of miR-217 has also been found to be upregulated in gastric and breast cancer $(42,43)$. These findings indicated that miR-217 functions as both an oncomir and tumor-suppressive miRNA, depending on the tumor type. Notably, several studies have reported that miR-217 was increased in HCC $(44,45)$, however the specific mechanisms responsible for the progression of HCC remain poorly elucidated. Consistent with these findings, we revealed that miR-217 was upregulated in HCC tissues and cells. Overexpression of miR-217 promoted, while silencing miR-217 suppressed, the CSC-like phenotypes in vitro and tumorigenicity in vivo in $\mathrm{HCC}$ cells. Our findings further demonstrated that miR-217 promotes the CSC-like phenotype via DKK1 targeting, resulting in constitutive activation of Wnt signaling. Moreover, the stimulatory or inhibitory effects of the upregulation or downregulation of miR-217 on stem cell properties and Wnt signaling were antagonized by the upregulation or silencing of DKK1 in HCC cells. Notably, another study reported that miR-217 expression was much lower in highly invasive MHCC-97H HCC cells and metastatic HCC tissues. Ectopic expression of miR-217 inhibited the invasion of MHCC-97H cells. Inversely, inhibition of miR-217 enhanced the invasive ability of Huh7 and MHCC-97L cells (46). This evidence demonstrated that miR-217 plays different or even contradicting roles in the different developmental processes of HCC.

In summary, the present study demonstrated that oncogenic miR-217 promotes CSC-like properties and tumorigenicity by negatively regulating DKK1, leading to activation of the Wnt/ $\beta$-catenin signaling pathway in HCC. Improved understanding of the specific role of miR-217 in the activation of the Wnt signaling pathway and in the pathogenesis of HCC may help to develop novel therapeutic methods in the treatment of HCC.

\section{Acknowledgements}

The present study was supported by the State Key Program of the National Natural Science Foundation of Guangdong, China (Program, no. 2015A030311039), the National Natural Science Foundation of China (81272312) and the Science and Technology Planning Project of Guangdong Province, China (no. 2014A020212390).

\section{References}

1. Fong Y, Sun RL, Jarnagin W and Blumgart LH: An analysis of 412 cases of hepatocellular carcinoma at a Western center. Ann Surg 229: 790-800, 1999 .
2. Gerber B, Freund M and Reimer T: Recurrent breast cancer: Treatment strategies for maintaining and prolonging good quality of life. Dtsch Arztebl Int 107: 85-91, 2010.

3. Clarke MF, Dick JE, Dirks PB, Eaves CJ, Jamieson CH, Jones DL, Visvader J, Weissman IL and Wahl GM: Cancer stem cells - perspectives on current status and future directions: AACR Workshop on cancer stem cells. Cancer Res 66: 9339-9344, 2006.

4. Visvader JE and Lindeman GJ: Cancer stem cells in solid tumours: Accumulating evidence and unresolved questions. Nat Rev Cancer 8: 755-768, 2008.

5. Chaffer CL and Weinberg RA: A perspective on cancer cell metastasis. Science 331: 1559-1564, 2011.

6. Monteiro $\mathrm{J}$ and Fodde R: Cancer stemness and metastasis: Therapeutic consequences and perspectives. Eur J Cancer 46: 1198-1203, 2010

7. Lapidot T, Sirard C, Vormoor J, Murdoch B, Hoang T, CaceresCortes J, Minden M, Paterson B, Caligiuri MA and Dick JE: A cell initiating human acute myeloid leukaemia after transplantation into SCID mice. Nature 367: 645-648, 1994.

8. O'Brien CA, Pollett A, Gallinger S and Dick JE: A human colon cancer cell capable of initiating tumour growth in immunodeficient mice. Nature 445: 106-110, 2007.

9. Hermann PC, Huber SL, Herrler T, Aicher A, Ellwart JW, Guba M, Bruns CJ and Heeschen C: Distinct populations of cancer stem cells determine tumor growth and metastatic activity in human pancreatic cancer. Cell Stem Cell 1: 313-323, 2007.

10. Al-Hajj M, Wicha MS, Benito-Hernandez A, Morrison SJ and Clarke MF: Prospective identification of tumorigenic breast cancer cells. Proc Natl Acad Sci USA 100: 3983-3988, 2003.

11. Lee TK, Castilho A, Cheung VC, Tang KH, Ma S and Ng IO: $\mathrm{CD} 24^{+}$liver tumor-initiating cells drive self-renewal and tumor initiation through STAT3-mediated NANOG regulation. Cell Stem Cell 9: 50-63, 2011.

12. Zhao W, Wang L, Han H, Jin K, Lin N, Guo T, Chen Y, Cheng H, $\mathrm{Lu} \mathrm{F}$, Fang W, et al: 1B50-1, a mAb raised against recurrent tumor cells, targets liver tumor-initiating cells by binding to the calcium channel $\alpha 2 \delta 1$ subunit. Cancer Cell 23: 541-556, 2013.

13. Inui M, Martello $\mathrm{G}$ and Piccolo S: MicroRNA control of signal transduction. Nat Rev Mol Cell Biol 11: 252-263, 2010.

14. Bartel DP: MicroRNAs: Genomics, biogenesis, mechanism, and function. Cell 116: 281-297, 2004.

15. Ren D, Wang M, Guo W, Huang S, Wang Z, Zhao X, Du H, Song L and Peng X: Double-negative feedback loop between ZEB2 and miR-145 regulates epithelial-mesenchymal transition and stem cell properties in prostate cancer cells. Cell Tissue Res 358: 763-778, 2014.

16. Ren D, Wang M, Guo W, Zhao X, Tu X, Huang S, Zou X and Peng X: Wild-type p53 suppresses the epithelial-mesenchymal transition and stemness in PC-3 prostate cancer cells by modulating miR-145. Int J Oncol 42: 1473-1481, 2013.

17. Ma S, Tang KH, Chan YP, Lee TK, Kwan PS, Castilho A, Ng I, Man K, Wong N, To KF, et al: miR-130b promotes CD133+ liver tumor-initiating cell growth and self-renewal via tumor protein 53-induced nuclear protein 1. Cell Stem Cell 7: 694-707, 2010.

18. Ji J, Yamashita T, Budhu A, Forgues M, Jia HL, Li C, Deng C, Wauthier E, Reid LM, Ye QH, et al: Identification of microRNA-181 by genome-wide screening as a critical player in EpCAM-positive hepatic cancer stem cells. Hepatology 50: 472-480, 2009.

19. Hahn WC, Dessain SK, Brooks MW, King JE, Elenbaas B, Sabatini DM, DeCaprio JA and Weinberg RA: Enumeration of the simian virus 40 early region elements necessary for human cell transformation. Mol Cell Biol 22: 2111-2123, 2002.

20. Guo W, Ren D, Chen X, Tu X, Huang S, Wang M, Song L, Zou X and Peng X: HEF1 promotes epithelial mesenchymal transition and bone invasion in prostate cancer under the regulation of microRNA-145. J Cell Biochem 114: 1606-1615, 2013.

21. Wang M, Ren D, Guo W, Huang S, Wang Z, Li Q, Du H, Song L and Peng X: N-cadherin promotes epithelial-mesenchymal transition and cancer stem cell-like traits via ErbB signaling in prostate cancer cells. Int J Oncol 48: 595-606, 2016.

22. Li J, Gong LY, Song LB, Jiang LL, Liu LP, Wu J, Yuan J, Cai JC, $\mathrm{He}$ M, Wang L, et al: Oncoprotein Bmi-1 renders apoptotic resistance to glioma cells through activation of the IKK-nuclear factor-kappaB pathway. Am J Pathol 176: 699-709, 2010.

23. Goodell MA: Stem cell identification and sorting using the Hoechst 33342 side population (SP). Curr Protoc Cytom Chapter 9: Unit9.18, 2005. 
24. de Sousa E Melo F, Colak S, Buikhuisen J, Koster J, Cameron K, de Jong JH, Tuynman JB, Prasetyanti PR, Fessler E, van den Bergh SP, et al: Methylation of cancer-stem-cellassociated Wnt target genes predicts poor prognosis in colorectal cancer patients. Cell Stem Cell 9: 476-485, 2011.

25. Simeone DM: Pancreatic cancer stem cells: Implications for the treatment of pancreatic cancer. Clin Cancer Res 14: 5646-5648, 2008.

26. Mao J, Wang J, Liu B, Pan W, Farr GH III, Flynn C, Yuan H, Takada S, Kimelman D, Li L, et al: Low-density lipoprotein receptor-related protein-5 binds to Axin and regulates the canonical Wnt signaling pathway. Mol Cell 7: 801-809, 2001.

27. Gordon MD and Nusse R: Wnt signaling: Multiple pathways, multiple receptors, and multiple transcription factors. J Biol Chem 281: 22429-22433, 2006.

28. Barker N, van Es JH, Kuipers J, Kujala P, van den Born M, Cozijnsen M, Haegebarth A, Korving J, Begthel H, Peters PJ, et al: Identification of stem cells in small intestine and colon by marker gene Lgr5. Nature 449: 1003-1007, 2007.

29. Wielenga VJ, Smits R, Korinek V, Smit L, Kielman M, Fodde R, Clevers $\mathrm{H}$ and Pals ST: Expression of CD44 in $A p c$ and Tcf mutant mice implies regulation by the WNT pathway. Am J Pathol 154: 515-523, 1999.

30. Gangopadhyay S, Nandy A, Hor P and Mukhopadhyay A: Breast cancer stem cells: A novel therapeutic target. Clin Breast Cancer 13: 7-15, 2013

31. Takebe N, Miele L, Harris PJ, Jeong W, Bando H, Kahn M, Yang SX and Ivy SP: Targeting Notch, Hedgehog, and Wnt pathways in cancer stem cells: Clinical update. Nat Rev Clin Oncol 12: 445-464, 2015.

32. Lachenmayer A, Alsinet C, Savic R, Cabellos L, Toffanin S, Hoshida Y, Villanueva A, Minguez B, Newell P, Tsai HW, et al: Wnt-pathway activation in two molecular classes of hepatocellular carcinoma and experimental modulation by sorafenib. Clin Cancer Res 18: 4997-5007, 2012.

33. Wong CM, Fan ST and Ng IO: beta-Catenin mutation and overexpression in hepatocellular carcinoma: Clinicopathologic and prognostic significance. Cancer 92: 136-145, 2001.

34. Bafico A, Liu G, Yaniv A, Gazit A and Aaronson SA: Novel mechanism of Wnt signalling inhibition mediated by Dickkopf-1 interaction with LRP6/Arrow. NaT cell Biol 3: 683-686, 2001.

35. Lee J, Yoon YS and Chung JH: Epigenetic silencing of the WNT antagonist DICKKOPF-1 in cervical cancer cell lines. Gynecol Oncol 109: 270-274, 2008.

36. Na Y, Lee SM, Kim DS and Park JY: Promoter methylation of Wnt antagonist DKK1 gene and prognostic value in Korean patients with non-small cell lung cancers.Cancer Biomark 12: 73-79, 2012.
37. Agur Z, Kirnasovsky OU, Vasserman G, Tencer-Hershkowicz L, Kogan Y, Harrison H, Lamb R and Clarke RB: Dickkopf1 regulates fate decision and drives breast cancer stem cells to differentiation: An experimentally supported mathematical model. PLoS One 6: e24225, 2011.

38. Kim Y, Kim H, Park D, Han M, Lee H, Lee YS, Choe J, Kim YM and Jeoung D: miR-217 and CAGE form feedback loop and regulates the response to anti-cancer drugs through EGFR and HER2. Oncotarget 7: 10297-10321, 2016.

39. Rachagani S, Macha MA, Menning MS, Dey P, Pai P, Smith LM, Mo YY and Batra SK: Changes in microRNA (miRNA) expression during pancreatic cancer development and progression in a genetically engineered $\mathrm{Kras}^{\mathrm{G} 12 \mathrm{D}}$;Pdx1-Cre mouse (KC) model. Oncotarget 6: 40295-40309, 2015.

40. Gu L, Li H, Chen L, Ma X, Gao Y, Li X, Zhang Y, Fan Y and Zhang X: MicroRNAs as prognostic molecular signatures in renal cell carcinoma: A systematic review and meta-analysis. Oncotarget 6: 32545-32560, 2015.

41. Chen DL, Zhang DS, Lu YX, Chen LZ, Zeng ZL, He MM, Wang FH, Li YH, Zhang HZ, Pelicano H, et al: microRNA-217 inhibits tumor progression and metastasis by downregulating EZH2 and predicts favorable prognosis in gastric cancer. Oncotarget 6: 10868-10879, 2015.

42. Yang M, Cui G, Ding M, Yang W, Liu Y, Dai 1 and Chen L: miR-935 promotes gastric cancer cell proliferation by targeting SOX7. Biomed Pharmacother 79: 153-158, 2016.

43. Zhang AX, Lu FQ, Yang YP, Ren XY, Li ZF and Zhang W: MicroRNA-217 overexpression induces drug resistance and invasion of breast cancer cells by targeting PTEN signaling. Cell Biol Int: Jun 24, 2015 (Epub ahead of print). doi: 10.1002/cbin.10506.

44. Wang W, Zhao LJ, Tan YX, Ren H and Qi ZT: Identification of deregulated miRNAs and their targets in hepatitis B virus-associated hepatocellular carcinoma. World J Gastroenterol 18: 5442-5453, 2012

45. Wang W, Zhao LJ, Tan YX, Ren H and Qi ZT: MiR-138 induces cell cycle arrest by targeting cyclin D3 in hepatocellular carcinoma. Carcinogenesis 33: 1113-1120, 2012.

46. Su J, Wang Q, Liu Y and Zhong M: miR-217 inhibits invasion of hepatocellular carcinoma cells through direct suppression of E2F3. Mol Cell Biochem 392: 289-296, 2014. 